\title{
Research Article \\ On Generalized Implicit Vector Equilibrium Problems in Topological Ordered Spaces
}

\author{
Qun Luo \\ Department of Mathematics, Zhaoqing University, Zhaoqing, Guangdong 526061, China \\ Correspondence should be addressed to Qun Luo, liluoqun@yahoo.com.cn \\ Received 4 June 2009; Revised 29 August 2009; Accepted 30 September 2009 \\ Recommended by Nanjing Jing Huang \\ We discuss three classes of generalized implicit vector equilibrium problems in topological ordered \\ spaces. Under some conditions, we prove three new existence theorems of solutions for the \\ generalized implicit vector equilibrium problems in topological ordered spaces by using the Fan- \\ Browder fixed point theorem. \\ Copyright (C) 2009 Qun Luo. This is an open access article distributed under the Creative Commons \\ Attribution License, which permits unrestricted use, distribution, and reproduction in any \\ medium, provided the original work is properly cited.
}

\section{Introduction and Preliminaries}

It is well known that the vector equilibrium problem is closely related to vector variational inequality, vector optimization problem, and many others (see, e.g., [1-6] and the references therein).

Recently, a large of generalized vector equilibrium problems have been studied in different conditions by many authors and a lot of results concerned with the existence of solutions and properties of solutions have been given in finite and infinite dimensional spaces (see [7] and the references therein).

The main purpose of this paper is to extend some known results for vector equilibrium problems to topological ordered spaces (see [8]). We discuss three classes of generalized implicit vector equilibrium problems in topological ordered spaces. Under some conditions, we prove three new existence theorems of solutions for the generalized implicit vector equilibrium problems in topological ordered spaces by using the Fan-Browder fixed point theorem.

A semilattice is a partially ordered set $X$, with the partial ordering denoted by $\leq$, for which any pair $\left(x, x^{\prime}\right)$ of elements has a least upper bound, denoted by $x \vee x^{\prime}$. It is easy to see that any nonempty finite subset $A$ of $X$ has a least upper bound, denoted by sup $A$. In the 
case $x \leq x^{\prime}$, the set $\left[x, x^{\prime}\right]=\left\{y \in X: x \leq y \leq x^{\prime}\right\}$ is called an order interval. Now assume that $(X, \leq)$ is a semilattice and $A \subseteq X$ is a nonempty finite subset. Thus, the set

$$
\Delta(A)=\bigcup_{a \in A}[a, \sup A]
$$

is well defined and it has the following properties:

(a) $A \subseteq \Delta(A)$,

(b) if $A \subseteq A^{\prime}$, then $\Delta(A) \subseteq \Delta\left(A^{\prime}\right)$.

A subset $E \subseteq X$ is said to be $\Delta$-convex if, for any nonempty finite subset $A \subseteq E$, we have $\Delta(A) \subseteq E$.

For any $D \subset X, F(D)$ denotes the family of all finite subsets of $D$ and

$$
\Delta(D)=\bigcup_{A \in \mathcal{F}(D)} \Delta(A)
$$

Let $X$ be a topological semilattice, $K \subset X$ a nonempty $\Delta$-convex subset, $Y$ a Hausdorff topological vector space. Assume that $A: K \rightarrow 2^{K}, g: K \rightarrow 2^{K}, f: K \times K \rightarrow 2^{Y}$, and $C: K \rightarrow 2^{Y}$ such that, for any $x \in K, C(x)$ is a closed, pointed, and convex cone in $Y$ and $\operatorname{int} C(x) \neq \emptyset$.

In this paper, we consider the following three classes generalized implicit vector equilibrium problems:

(1) weak generalized implicit vector equilibrium problem (WGIVEP): find $x^{*} \in K$, such that $x^{*} \in A\left(x^{*}\right)$ and for any $y \in A\left(x^{*}\right)$, there exists an $u \in g\left(x^{*}\right)$ such that

$$
f(u, y) \cap \operatorname{int} C\left(x^{*}\right)=\emptyset
$$

(2) strong generalized implicit vector equilibrium problem (SGIVEP): find $x^{*} \in K$ such that $x^{*} \in A\left(x^{*}\right)$ and

$$
f(u, y) \cap \operatorname{int} C\left(x^{*}\right)=\emptyset, \quad \forall y \in A\left(x^{*}\right), \forall u \in g\left(x^{*}\right) .
$$

(3) uniform generalized implicit vector equilibrium problem (UGIVEP): find $x^{*} \in K$ such that $x^{*} \in A\left(x^{*}\right)$ and there exists $u^{*} \in g\left(x^{*}\right)$ such that

$$
f\left(u^{*}, y\right) \cap C\left(x^{*}\right)=\emptyset, \quad \forall y \in A\left(x^{*}\right)
$$

Definition 1.1. Let $X$ and $Y$ be two topological spaces.

(1) A mapping $F: X \rightarrow 2^{Y}$ is called upper semicontinuous (usc) at $x_{0} \in X$ if, for any neighborhood $N\left(F\left(x_{0}\right)\right)$ of $F\left(x_{0}\right)$, there exists a neighborhood $N\left(x_{0}\right)$ of $x_{0}$ such that

$$
F(x) \subset N\left(F\left(x_{0}\right)\right), \quad \forall x \in N\left(x_{0}\right) .
$$

$F$ is called usc on $X$ if it is usc at each point of $X$. 
(2) A mapping $F: X \rightarrow 2^{\gamma}$ is called lower semicontinuous (lsc) at $x_{0} \in X$ if, for any net $\left\{x_{\alpha}\right\}$ in $X$ such that $x_{\alpha} \rightarrow x_{0}$ and for any $y_{0} \in F\left(x_{0}\right)$, there exists $y_{\alpha} \in F\left(x_{\alpha}\right)$ such that $y_{\alpha} \rightarrow y_{0} . F$ is called lsc on $X$ if it is lsc at each point of $X$.

(3) A mapping $F: X \rightarrow 2^{Y}$ is called complement pseudo-upper semicontinuous (c p-usc) at $x_{0} \in X$ if, for $y \notin F\left(x_{\alpha}\right)$ with $x_{\alpha} \rightarrow x_{0}$, we have $y \notin F\left(x_{0}\right)$. $F$ is called c p-usc on $X$ if $F$ is c p-usc at each point $x_{0}$ of $X$.

Remark 1.2. (1) By [9], if $F$ is usc with closed values, then for any net $\left\{x_{\alpha}\right\}$ in $X$ such that $x_{\alpha} \rightarrow x_{0}$ and for any net $\left\{y_{\alpha}\right\}$ in $Y$ with $y_{\alpha} \in F\left(x_{\alpha}\right)$ such that $y_{\alpha} \rightarrow y_{0}$ in $Y$, we have $y_{0} \in F\left(x_{0}\right)$.

(2) If $Y \backslash F$ is usc with closed values, then $F$ is c p-usc, where

$$
(Y \backslash F)(x)=Y \backslash F(x), \quad \forall x \in X
$$

Lemma 1.3 (see [9]). Let $X$ and $Y$ be two topological spaces. Let $X$ be compact and $F: X \rightarrow 2^{Y}$ be usc such that $F(x)$ is compact for each $x \in X$. Then $F(X)=\bigcup_{x \in X} F(x)$ is compact.

Definition 1.4. Let $X$ be a topological semilattice or a $\Delta$-convex subset of a topological semilattice, let $Y$ be a Hausdorff topological vector space, and let $C \subset Y$ be a closed, pointed, and convex cone with int $C \neq \emptyset$.

(1) A mapping $F: X \rightarrow 2^{Y}$ is called a $\Delta$-convex mapping (or a $\Delta$-concave mapping) with respect to $C$ if, for any nonempty finite subset $D=\left\{x_{1}, x_{2}, \ldots, x_{n}\right\} \subseteq X, x \in \Delta(D)$, $z_{i} \in F\left(x_{i}\right), t_{i} \geq 0$ with $i=1,2, \ldots, n$ and $\sum_{i=1}^{n} t_{i}=1$, there exists $z \in F(x)$ such that

$$
\sum_{i=1}^{n} t_{i} z_{i}-z \in C\left(\text { or } z-\sum_{i=1}^{n} t_{i} z_{i} \in C\right)
$$

(2) A mapping $F: X \rightarrow 2^{\gamma}$ is called to have $\Delta$-inheritance if, for any nonempty finite subset $D=\left\{x_{1}, x_{2}, \ldots, x_{n}\right\}$ of $X, x_{0} \in \Delta(D), K \subset X$ with $K \cap F\left(x_{i}\right)=\emptyset$ and $i=1,2, \ldots, n$, we have $K \cap F\left(x_{0}\right)=\emptyset$.

(3) Let $Q \subseteq X$. A mapping $G: X \times X \rightarrow 2^{Y}$ is called $Q$ - $\Delta$-convex (or $Q$ - $\Delta$-concave) with respect to $C$ in second argument if, for any nonempty finite subset $D=\left\{x_{1}, x_{2}, \ldots, x_{n}\right\} \subseteq X$, $x \in \Delta(D),\left\{u_{0}, u_{1}, u_{2}, \ldots, u_{n}\right\} \subseteq Q, z_{i} \in G\left(u_{i}, x_{i}\right), t_{i} \geq 0$ with $i=1,2, \ldots, n$ and $\sum_{i=1}^{n} t_{i}=1$, there exists $z \in G\left(u_{0}, x\right)$ such that

$$
\sum_{i=1}^{n} t_{i} z_{i}-z \in C\left(\text { or } z-\sum_{i=1}^{n} t_{i} z_{i} \in C\right)
$$

Remark 1.5. If $G: X \times X \rightarrow 2^{Y}$ is a $Q-\Delta$-convex mapping (or a $Q$ - $\Delta$-concave mapping) with respect to $C$ in second argument, then for any $u \in X, G(u, \cdot): X \rightarrow 2^{Y}$ is a $\Delta$-convex mapping (or a $\Delta$-concave mapping) with respect to $C$.

Lemma 1.6 (see [10]). Let $K$ be a nonempty compact $\Delta$-convex subset of a topological semilattice with path-connected intervals $M$, let $F: K \rightarrow 2^{K}$ be a mapping with nonempty $\Delta$-convex values such that, for each $y \in K, F^{-1}(y)$ is an open set in $K$. Then $F$ has a fixed point. 


\section{Existence Theorems}

Theorem 2.1. Let $K$ be a nonempty compact $\Delta$-convex subset of a topological semilattice with pathconnected intervals $M$, let $Y$ be a Hausdorff topological vector space. Let $A: K \rightarrow 2^{K}$ be a mapping with nonempty $\Delta$-convex values, and let $g: K \rightarrow 2^{K}$ and $f: K \times K \rightarrow 2^{Y}$ be mappings and $C: K \rightarrow 2^{Y}$ be a mapping such that, for each $x \in K, C(x)$ is a closed, pointed, and convex cone in $Y$ with int $C(x) \neq \emptyset$. Assume that

(1) For any $y \in K, A^{-1}(y)$ is open;

(2) $B=\{x \in K: x \in A(x)\}$ is closed;

(3) $g$ is usc with compact values;

(4) $f(u, x) \cap \operatorname{int} C(x)=\emptyset$ for any $x \in K$ and $u \in g(x)$;

(5) for any $x \in K$ and $u \in g(x), y \rightarrow f(u, y)$ is $\Delta$-concave with respect to $C(x)$;

(6) for any $y \in K, x \rightarrow f(x, y)$ is lsc;

(7) $W: K \rightarrow 2^{Y}$ is usc, where $W(x)=Y \backslash \operatorname{int} C(x)$ for each $x \in K$.

Then there exists an $x^{*} \in K$ such that $x^{*} \in A\left(x^{*}\right)$ and for any $y \in A\left(x^{*}\right)$, there exists an $u^{*} \in g\left(x^{*}\right)$ such that

$$
f\left(u^{*}, y\right) \cap \operatorname{int} C\left(x^{*}\right)=\emptyset .
$$

Furthermore, the solution set of (WGIVEP) is closed, and hence is compact.

Proof. Define $P: K \rightarrow 2^{K}$ by

$$
P(x)=\{y \in K: \forall u \in g(x), f(u, y) \cap \operatorname{int} C(x) \neq \emptyset\}, \quad \forall x \in K .
$$

We first prove that for any $y \in K, P^{-1}(y)$ is open, that is,

$$
\begin{aligned}
K \backslash P^{-1}(y) & =\left\{x \in K: x \notin P^{-1}(y)\right\} \\
& =\{x \in K: y \notin P(x)\} \\
& =\{x \in K: \exists u \in g(x), f(u, y) \cap \operatorname{int} C(x)=\emptyset\}
\end{aligned}
$$

is closed. Let a net $\left\{x_{\alpha}\right\} \subset K \backslash P^{-1}(y)$ and $x_{\alpha} \rightarrow x_{0} \in K$. Then there exists $u_{\alpha} \in g\left(x_{\alpha}\right)$ such that $f\left(u_{\alpha}, y\right) \cap \operatorname{int} C\left(x_{\alpha}\right)=\emptyset, f\left(u_{\alpha}, y\right) \subset W\left(x_{\alpha}\right)$, for any $\alpha$. By (3) and Lemma 1.3, we know that

$$
g(K)=\bigcup_{x \in K} g(x)
$$

is compact and so $\left\{u_{\alpha}\right\} \subset g(K)$ has a cluster point $u_{0} \in g(K)$. We may assume that $u_{\alpha} \rightarrow u_{0}$ and thus, $u_{0} \in g\left(x_{0}\right)$. For any $v_{0} \in f\left(u_{0}, y\right)$, by (6), there exists $v_{\alpha} \in f\left(u_{\alpha}, y\right)$ such that $v_{\alpha} \rightarrow v_{0}$ and so $v_{\alpha} \in f\left(u_{\alpha}, y\right) \subset W\left(x_{\alpha}\right)$. It follows from (7) that $v_{0} \in W\left(x_{0}\right)=Y \backslash \operatorname{int} C\left(x_{0}\right)$ and hence $f\left(u_{0}, y\right) \cap \operatorname{int} C\left(x_{0}\right)=\emptyset$. Thus, $K \backslash P^{-1}(y)$ is closed and so $P^{-1}(y)$ is open. 
Suppose that there exists an $x_{0} \in K$ such that $P\left(x_{0}\right)$ is not $\Delta$-convex, that is, there exist $y_{1}, y_{2}, \ldots, y_{n} \in P\left(x_{0}\right)$ such that $\Delta\left(y_{1}, y_{2}, \ldots, y_{n}\right) \not \subset P\left(x_{0}\right)$. Hence, there exists $y_{0} \in$ $\Delta\left(y_{1}, y_{2}, \ldots, y_{n}\right), y_{0} \notin P\left(x_{0}\right)$, that is, there exists $u_{0} \in g\left(x_{0}\right)$ such that $f\left(u_{0}, y_{0}\right) \cap \operatorname{int} C\left(x_{0}\right)=$ $\emptyset$. For each $i=1,2, \ldots, n, y_{i} \in P\left(x_{0}\right)$, take $u=u_{0}, f\left(u_{0}, y_{i}\right) \cap \operatorname{int} C\left(x_{0}\right) \neq \emptyset$. Let $v_{i} \in$ $f\left(u_{0}, y_{i}\right) \cap \operatorname{int} C\left(x_{0}\right), i=1,2, \ldots, n$. For any $t_{i} \geq 0, i=1,2, \ldots, n$, and $\sum_{i=1}^{n} t_{i}=1$, we have $\sum_{i=1}^{n} t_{i} v_{i} \in \operatorname{int} C\left(x_{0}\right)$. By (5), there exists $v \in f\left(u_{0}, y_{0}\right)$ such that

$$
v-\sum_{i=1}^{n} t_{i} v_{i} \in C\left(x_{0}\right)
$$

Since $v \notin \operatorname{int} C\left(x_{0}\right)$, we know that

$$
v-\left(v-\sum_{i=1}^{n} t_{i} v_{i}\right)=\sum_{i=1}^{n} t_{i} v_{i} \notin \operatorname{int} C\left(x_{0}\right),
$$

which is a contradiction. Therefore, for any $x \in K, P(x)$ is $\Delta$-convex.

By (1) and Lemma 1.6, $B \neq \emptyset$. Define $S: K \rightarrow 2^{K}$ by

$$
S(x)= \begin{cases}A(x) \cap P(x), & \text { if } \mathrm{x} \in \mathrm{B}, \\ A(x), & \text { if } \mathrm{x} \in \mathrm{K} \backslash \mathrm{B} .\end{cases}
$$

Then $S(x)$ is $\Delta$-convex for each $x \in K$. It follows from (1) and (2) that

$$
S^{-1}(y)=\left(A^{-1}(y) \cap P^{-1}(y)\right) \cup\left((K \backslash B) \cap A^{-1}(y)\right), \quad \forall y \in K
$$

is open.

Suppose that for all $x \in K, S(x)$ is nonempty. Then, by Lemma $1.6 S$ has a fixed point, that is, there exists $x_{0} \in K$, such that $x_{0} \in S\left(x_{0}\right)$. If $x_{0} \in B$, then $x_{0} \in S\left(x_{0}\right)=A\left(x_{0}\right) \cap P\left(x_{0}\right)$, hence $x_{0} \in P\left(x_{0}\right)$, for all $u \in g\left(x_{0}\right), f\left(u, x_{0}\right) \cap$ int $C\left(x_{0}\right) \neq \emptyset$ which contradicts to assumption (4); If $x_{0} \in K \backslash B$, then $x_{0} \in S\left(x_{0}\right)=A\left(x_{0}\right)$, hence $x_{0} \in B$ which contradicts with $x_{0} \in K \backslash B$. Therefore, there exists $x^{*} \in K$, such that $S\left(x^{*}\right)=\emptyset$. Since $A(x)$ is nonempty for any $x \in K$, then $x^{*} \in B, S\left(x^{*}\right)=A\left(x^{*}\right) \cap P\left(x^{*}\right)=\emptyset$, that is, $x^{*} \in A\left(x^{*}\right)$ and for any $y \in A\left(x^{*}\right), y \notin P\left(x^{*}\right)$. Therefore, $x^{*} \in A\left(x^{*}\right)$ and for any $y \in A\left(x^{*}\right)$, there exists an $u^{*} \in g\left(x^{*}\right)$ such that

$$
f\left(u^{*}, y\right) \cap \operatorname{int} C\left(x^{*}\right)=\emptyset .
$$

Let $T$ denote the solution set of (WGIVEP) and $\left\{x_{\alpha}\right\} \subset T$ with $x_{\alpha} \rightarrow x_{0} \in K$. We show that $x_{0} \in T$, that is, $x_{0} \in A\left(x_{0}\right)$, and for all $y \in A\left(x_{0}\right)$, there exists $u_{0} \in g\left(x_{0}\right)$ such that

$$
f\left(u_{0}, y\right) \cap \operatorname{int} C\left(x_{0}\right)=\emptyset .
$$

In fact, it follows from (2) that $x_{0} \in A\left(x_{0}\right)$. For any $y \in A\left(x_{0}\right), x_{0} \in A^{-1}(y)$. By (1), there exists an open neighborhood $N\left(x_{0}\right)$ of $x_{0}$ such that $N\left(x_{0}\right) \subset A^{-1}(y)$. Since $x_{\alpha} \rightarrow x_{0}$, there 
exists $\alpha_{0}$ such that for any $\alpha \geq \alpha_{0}, x_{\alpha} \in N\left(x_{0}\right) \subset A^{-1}(y)$. Thus, $y \in A\left(x_{\alpha}\right)$ and so there exists $u_{\alpha} \in g\left(x_{\alpha}\right)$ such that $f\left(u_{\alpha}, y\right) \cap \operatorname{int} C\left(x_{\alpha}\right)=\emptyset$, that is, $f\left(u_{\alpha}, y\right) \subset W\left(x_{\alpha}\right)$. Since $g(K)=\bigcup_{x \in K} g(x)$ is compact, $\left\{u_{\alpha}\right\} \subset g(K)$ has a cluster point $u_{0} \in g(K)$. We may assume that $u_{\alpha} \rightarrow u_{0}$. From (3), we have $u_{0} \in g\left(x_{0}\right)$. By (6), for any $q \in f\left(u_{0}, y\right)$, there exists $q_{\alpha} \in f\left(u_{\alpha}, y\right)$ such that $q_{\alpha} \rightarrow q$. It follows from (7) that $q \in W\left(x_{0}\right)$, that is, $f\left(u_{0}, y\right) \cap \operatorname{int} C\left(x_{0}\right)=\emptyset$. Thus, $T$ is closed, and hence is compact. This completes the proof.

Theorem 2.2. Let $K$ be a nonempty compact $\Delta$-convex subset of a topological semilattice with pathconnected intervals $M$, let $Y$ be a Hausdorff topological vector space. Let $A: K \rightarrow 2^{K}$ be with nonempty $\Delta$-convex values, and let $g: K \rightarrow 2^{K}$ and $f: K \times K \rightarrow 2^{Y}$ be mappings and $C: K \rightarrow$ $2^{Y}$ be a mapping such that, for each $x \in K, C(x)$ is a closed, pointed, and convex cone in $Y$ with $\operatorname{int} C(x) \neq \emptyset$. Assume that

(1) For any $y \in K, A^{-1}(y)$ is open;

(2) $B=\{x \in K: x \in A(x)\}$ is closed;

(3) $g$ is $l s c$;

(4) for all $x \in K$ and $u \in g(x), f(u, x) \cap \operatorname{int} C(x)=\emptyset$;

(5) for all $x \in K, f$ is $g(x)$ - $\Delta$-concave with respect to $C(x)$ in second argument;

(6) for all $y \in K, x \rightarrow f(x, y)$ is lsc;

(7) $W: K \rightarrow 2^{Y}$ is usc, where $W(x)=Y \backslash \operatorname{int} C(x)$ for all $x \in K$.

Then there exists an $x^{*} \in K$ such that $x^{*} \in A\left(x^{*}\right)$ and

$$
f(u, y) \cap \operatorname{int} C\left(x^{*}\right)=\emptyset, \quad \forall y \in A\left(x^{*}\right), \quad \forall u \in g\left(x^{*}\right) .
$$

Furthermore, the solution set of (SGIVEP) is closed, and hence is compact.

Proof. Define $P: K \rightarrow 2^{K}$ by

$$
P(x)=\{y \in K: \exists u \in g(x), f(u, y) \cap \operatorname{int} C(x) \neq \emptyset\}, \quad \forall x \in K .
$$

Then the proof is similar to that of Theorem 2.1 and so we omit it.

Theorem 2.3. Let $K$ be a nonempty compact $\Delta$-convex subset of a topological semilattice with pathconnected intervals $M$ and let $Y$ be a Hausdorff topological vector space. Let $A: K \rightarrow 2^{K}$ be with nonempty $\Delta$-convex values, let $g: K \rightarrow 2^{K}$ and $f: K \times K \rightarrow 2^{Y}$ be mappings, and let $C: K \rightarrow$ $2^{Y}$ be a mapping such that, for each $x \in K, C(x)$ is a closed, pointed, and convex cone in $Y$ with $\operatorname{int} C(x) \neq \emptyset$. Assume that

(1) $A$ is usc with compact values;

(2) For any $x \in K, g(x)$ is nonempty $\Delta$-convex;

(3) $g$ is c p-usc on $X$;

(4) $f$ is usc with nonempty compact values;

(5) for all $x, u \in K, f(u, x) \cap C(x)=\emptyset$;

(6) for all $u, y \in K, x \rightarrow f(u, x)$ is $\Delta$-concave with respect to $C(y)$; 
Fixed Point Theory and Applications

(7) C has $\Delta$-inheritance;

(8) $C$ is c p-usc on $X$.

Then there exists an $x^{*} \in K$ such that $x^{*} \in A\left(x^{*}\right)$ and there exists $u^{*} \in g\left(x^{*}\right)$ such that

$$
f\left(u^{*}, y\right) \cap C\left(x^{*}\right)=\emptyset, \quad \forall y \in A\left(x^{*}\right)
$$

Proof. Define $F: K \times K \rightarrow 2^{K \times K}$ by

$$
F(x, u)=B(x, u) \times g(x), \quad \forall(x, u) \in K \times K,
$$

where

$$
B(x, u)=\{y \in A(x): f(u, z) \cap C(y)=\emptyset, \forall z \in A(x)\}
$$

The proof is divided into the following five steps.

(I) For any $(x, u) \in K \times K, B(x, u)$ is nonempty.

If it is false, then there exists $(x, u) \in K \times K$ such that $B(x, u)=\emptyset$, that is, for any $y \in A(x)$, there exists $z \in A(x)$ such that $f(u, z) \cap C(y) \neq \emptyset$. Let

$$
G(y)=\{z \in A(x): f(u, z) \cap C(y) \neq \emptyset\} .
$$

Then $G: A(x) \rightarrow 2^{A(x)}$ is nonempty values. If there exists $y_{0} \in A(x)$ such that $G\left(y_{0}\right)$ is not $\Delta$ convex, then there exist $k_{1}, k_{2}, \ldots, k_{n} \in G\left(y_{0}\right)$ such that $\Delta\left(\left\{k_{1}, k_{2}, \ldots, k_{n}\right\}\right) \not \subset G\left(y_{0}\right)$, that is, there exists $q \in \Delta\left(\left\{k_{1}, k_{2}, \ldots, k_{n}\right\}\right)$ with $q \notin G\left(y_{0}\right)$. Thus, $f(u, q) \cap C\left(y_{0}\right)=\emptyset$. For each $i=1,2, \ldots, n$, $k_{i} \in G\left(y_{0}\right)$, take $v_{i} \in f\left(u, k_{i}\right) \cap C\left(y_{0}\right)$. For any $t_{i} \geq 0, i=1,2, \ldots, n$ and $\sum_{i=1}^{n} t_{i}=1$, we have $\sum_{i=1}^{n} t_{i} v_{i} \in C\left(y_{0}\right)$. By $(6)$, there exists $v \in f(u, q)$ such that

$$
v-\sum_{i=1}^{n} t_{i} v_{i} \in C\left(y_{0}\right)
$$

Since $f(u, q) \cap C\left(y_{0}\right)=\emptyset, v \notin C\left(y_{0}\right)$. Hence,

$$
v-\left(v-\sum_{i=1}^{n} t_{i} v_{i}\right)=\sum_{i=1}^{n} t_{i} v_{i} \notin C\left(y_{0}\right),
$$

which is a contradiction. Thus, for any $y \in A(x), G(y)$ is nonempty $\Delta$-convex.

For any $z \in A(x)$,

$$
G^{-1}(z)=\{y \in A(x): z \in G(y)\}=\{y \in A(x): f(u, z) \cap C(y) \neq \emptyset\} .
$$

It follows from (8) that

$$
A(x) \backslash G^{-1}(z)=\{y \in A(x): f(u, z) \cap C(y)=\emptyset\}
$$


is closed and so $G^{-1}(z)$ is open. Since $A(x)$ is nonempty compact and $\Delta$-convex, by Lemma $1.6 G$ has a fixed point. Thus, there exists $y^{*} \in A(x)$ such that $y^{*} \in G\left(y^{*}\right)$, that is, $f\left(u, y^{*}\right) \cap C\left(y^{*}\right) \neq \emptyset$ which contradicts with Assumption (5). Hence $B(x, u) \neq \emptyset$ for any $(x, u) \in K \times K$.

(II) For any $(x, u) \in K \times K, B(x, u)$ is $\Delta$-convex. If it is false, then there exists $(x, u) \in$ $K \times K$ such that $B(x, u)$ is not $\Delta$-convex, that is, there exist $y_{1}, y_{2}, \ldots, y_{n} \in B(x, u)$ such that

$$
\Delta\left(\left\{y_{1}, y_{2}, \ldots, y_{n}\right\}\right) \not \subset B(x, u)
$$

Thus, there exists $y_{0} \in \Delta\left(\left\{y_{1}, y_{2}, \ldots, y_{n}\right\}\right)$ such that $y_{0} \notin B(x, u)$. Then $y_{1}, y_{2}, \ldots, y_{n} \in A(x)$ and for all $z \in A(x), f(u, z) \cap C\left(y_{i}\right)=\emptyset, i=1,2, \ldots, n$. Since $A(x)$ is $\Delta$-convex, $y_{0} \in A(x)$. By (7), $f(u, z) \cap C\left(y_{0}\right)=\emptyset$. Since $y_{0} \notin B(x, u)$, there exists $z_{0} \in A(x)$ such that

$$
f\left(u, z_{0}\right) \cap C\left(y_{0}\right) \neq \emptyset,
$$

which is a contradiction. Therefore, for any $(x, u) \in K \times K, B(x, u)$ is $\Delta$-convex.

(III) $F(x, u)$ is nonempty $\Delta$-convex for any $(x, u) \in K \times K$. By steps (I) and (II), the conclusion follows directly from (2).

(IV) For any $(y, v) \in K \times K$,

$$
\begin{aligned}
F^{-1}(y, v) & =\{(x, u) \in K \times K:(y, v) \in F(x, u)\} \\
& =\{(x, u) \in K \times K: y \in B(x, u) \text { and } v \in g(x)\}
\end{aligned}
$$

is open. In fact, we only need to show that

$$
\begin{aligned}
(K \times K) \backslash F^{-1}(y, v) & =\{(x, u) \in K \times K:(y, v) \notin B(x, u) \times g(x)\} \\
& =\{(x, u) \in K \times K: y \notin B(x, u)\} \cup\{(x, u) \in K \times K: v \notin g(x)\}
\end{aligned}
$$

is closed. Let a net $\left\{\left(x_{\alpha}, u_{\alpha}\right)\right\} \subset\{(x, u) \in K \times K: y \notin B(x, u)\}$ and $\left(x_{\alpha}, u_{\alpha}\right) \rightarrow\left(x_{0}, u_{0}\right) \in K \times K$. If $y \notin A\left(x_{0}\right)$, then $y \notin B\left(x_{0}, u_{0}\right)$ and hence

$$
\left(x_{0}, u_{0}\right) \in\{(x, u) \in K \times K: y \notin B(x, u)\} .
$$

If $y \in A\left(x_{0}\right)$ and there exists $z_{\alpha} \in A\left(x_{\alpha}\right)$ such that

$$
f\left(u_{\alpha}, z_{\alpha}\right) \cap C(y) \neq \emptyset .
$$

Take $p_{\alpha} \in f\left(u_{\alpha}, z_{\alpha}\right) \cap C(y)$. By (1) and Lemma 1.3, $A(K)=\bigcup_{x \in K} A(x)$ is compact and hence $\left\{z_{\alpha}\right\} \subset A(K)$ has a cluster point $z_{0} \in A(K)$. We may assume that $z_{\alpha} \rightarrow z_{0}$ and so $z_{0} \in A\left(x_{0}\right)$. Similarly, by (4), $\left\{p_{\alpha}\right\}$ has a cluster point $p_{0}$. We assume that $p_{\alpha} \rightarrow p_{0}$ and hence $p_{0} \in f\left(u_{0}, z_{0}\right)$. Since $C(y)$ closed, $p_{0} \in C(y)$. Thus, $f\left(u_{0}, z_{0}\right) \cap C(y) \neq \emptyset, y \notin B\left(x_{0}, u_{0}\right)$, and $\left(x_{0}, u_{0}\right) \in\{(x, u) \in$ $K \times K: y \notin B(x, u)\}$. Hence, $\{(x, u) \in K \times K: y \notin B(x, u)\}$ is closed. Let a net

$$
\left\{\left(x_{\alpha}, u_{\alpha}\right)\right\} \subset\{(x, u) \in K \times K: v \notin g(x)\}
$$


and $\left(x_{\alpha}, u_{\alpha}\right) \rightarrow\left(x_{0}, u_{0}\right) \in K \times K$, then $v \notin g\left(x_{\alpha}\right)$. By (3), we have $v \notin g\left(x_{0}\right)$, and hence $\{(x, u) \in$ $K \times K: v \notin g(x)\}$ is closed. Thus, $(K \times K) \backslash F^{-1}(y, v)$ is closed and so $F^{-1}(y, v)$ is open.

(V) The UGIVEP has a solution. By Lemma 1.6, $F$ has a fixed point. Thus, there exists $\left(x^{*}, u^{*}\right) \in K \times K$ such that $\left(x^{*}, u^{*}\right) \in F\left(x^{*}, u^{*}\right)=B\left(x^{*}, u^{*}\right) \times g\left(x^{*}\right)$, that is, $x^{*} \in K$ and $u^{*} \in g\left(x^{*}\right)$ such that $x^{*} \in A\left(x^{*}\right)$ and

$$
f\left(u^{*}, y\right) \cap C\left(x^{*}\right)=\emptyset, \quad \forall y \in A\left(x^{*}\right) .
$$

This completes the proof.

\section{Acknowledgments}

This research was supported by the Natural Science Foundations of Guangdong Province (9251064101000015). The author is grateful to the referees for the valuable comments and suggestions.

\section{References}

[1] J. Li, N.-J. Huang, and J. K. Kim, “On implicit vector equilibrium problems,” Journal of Mathematical Analysis and Applications, vol. 283, no. 2, pp. 501-512, 2003.

[2] I. V. Konnov and J. C. Yao, "Existence of solutions for generalized vector equilibrium problems," Journal of Mathematical Analysis and Applications, vol. 233, no. 1, pp. 328-335, 1999.

[3] L.-C. Ceng, S.-M. Guu, and J.-C. Yao, "On generalized implicit vector equilibrium problems in Banach spaces," Computers E Mathematics with Applications, vol. 57, no. 10, pp. 1682-1691, 2009.

[4] X. H. Chen, "Existence of solution for the implicit multi-valued vector equilibrium problem," Journal of Applied Mathematics and Computing, vol. 30, no. 1-2, pp. 469-478, 2009.

[5] N. J. Huang, J. Li, and H. B. Thompson, "Implicit vector equilibrium problems with applications," Mathematical and Computer Modelling, vol. 37, no. 12-13, pp. 1343-1356, 2003.

[6] J. Li and N.-J. Huang, "Implicit vector equilibrium problems via nonlinear scalarisation," Bulletin of the Australian Mathematical Society, vol. 72, no. 1, pp. 161-172, 2005.

[7] G.-Y. Chen, X. X. Huang, and X. Q. Yang, Vector Optimization: Set-Valued and Variational Analysis, vol. 541 of Lecture Notes in Economics and Mathematical Systems, Springer, Berlin, Germany, 2005.

[8] C. D. Horvath and J. V. Llinares Ciscar, "Maximal elements and fixed points for binary relations on topological ordered spaces," Journal of Mathematical Economics, vol. 25, no. 3, pp. 291-306, 1996.

[9] J.-P. Aubin and I. Ekeland, Applied Nonlinear Analysis, Pure and Applied Mathematics, John Wiley \& Sons, New York, NY, USA, 1984.

[10] Q. Luo, "Ky Fan's section theorem and its applications in topological ordered spaces," Applied Mathematics Letters, vol. 17, no. 10, pp. 1113-1119, 2004. 